\title{
Caries experience by socio-behavioral characteristics in HIV-1 infected and uninfected Ugandan mothers - a multilevel analysis
}

NANCY BIRUNGI ( $\sim$ Nancy.Birungi@uib.no )

University of Bergen https://orcid.org/0000-0002-9071-196X

Lars Thore Fadnes

Universitetet i Bergen

Ingunn Marie Stadskleiv Engebretsen

Universitetet i Bergen

James Kashugyera Tumwine

Universitetet i Bergen

Stein Atle Lie

Universitetet i Bergen

Anne Nordrehaug Åstrøm

Universitetet i Bergen

Research article

Keywords: Dental caries, oral health, quality of life, HIV unexposed infected, children

Posted Date: July 16th, 2020

DOl: https://doi.org/10.21203/rs.3.rs-39656/v1

License: (c) (1) This work is licensed under a Creative Commons Attribution 4.0 International License.

Read Full License 


\section{Abstract}

Background: Few studies consider the oral health status of adults with HIV in comparison with negative controls. This study aims to assess caries experience in Ugandan mothers according to HIV status, sociobehavioral-characteristics and gingival bleeding status and to examine whether HIV status modifies the association of socio-behavioral characteristics with caries experience. Secondly, using multilevel analysis this study also assessed to what extent site specific caries experience vary between and within individuals.

Methods: This study uses data from the Ugandan site of the ANRS 121741 PROMISE- PEP trial (ClinicalTrials.gov, number NCT00640263) conducted in 2009-2013 that recruited mothers with HIV-1. In 2017 a comparison group of 181 HIV-1 negative controls was recruited. Caries experience was recorded using the World Health Organization's Decayed, Missed and Filled teeth/surfaces (DMFT/DMFS) indices. Mixed effects logistic regression with the melogit program within Stata was conducted with DMFT/DMFS as the outcome and HIV-1 infection status as well as socio-behavioral characteristics as exposures.

Results: Caries experience differed statistically significantly between HIV infected and non-infected mothers with $81 \%$ and $71 \%$ having DMFT $>0$, respectively. The corresponding mean and standard deviation were 4.6 (5.3) and 2.8 (3.2) respectively. Mixed effect logistic regression revealed significant positive associations between site specific caries and women's age (OR=1.8, 95\% Cl: 1.1-2.8), and presence of gingival bleeding ( $\mathrm{OR}=2.0,95 \% \mathrm{Cl}: 2.01 .2-3.2)$. Compared to premolar teeth, caries experience was more likely to occur in maxillary molars and mandibular molars and less likely to occur in incisors. The intra class correlation (ICC) for the correlation of individuals was 0.54 (95\% Cl: $0.48-0.59$ ). No two-way interactions terms between HIV-1 status and socio-demographic, behavioral and clinical covariates were observed.

Conclusion: The prevalence of dental caries is higher in Ugandan mothers with HIV-1 than in uninfected Ugandan mothers and varies with mothers', age, education and clinically recoded gingival inflammation. Socio-demographic differences in dental caries did not vary by HIV-1 status. We found that a multilevel random intercept model is appropriate and efficient for analyses of site specific dental caries data in the context of HIV-1 infected and uninfected Ugandan mothers

\section{Background}

Following widespread implementation of highly active anti-retroviral drugs (HAART), studies have shown an increase in life expectancy as well as in the prevalence of HIV-1 infected people worldwide [1]. Sub Saharan African countries are currently contributing $65 \%$ to the global total of 33 million people living with HIV [2]. Although people with HIV-1 are particularly vulnerable to oral diseases, studies examining their dental caries experience have so far been rare [3]. Some studies have reported increased dental caries prevalence in children with perinatally acquired HIV-1 infection [4-6]. It is unclear however, whether findings from those studies generalize to adult populations living with HIV /AIDS. 
Evidence suggests that HIV-1 infection under HAART influences the oral microbiome, thus emphasizing the vulnerability of individuals with HIV-1 to oral microbial diseases [7]. Studies have reported on strong associations between HIV-1 infection and oral mucosal lesions and periodontal disease [8-11]. Moreover, the prevalence of oral lesions seems to increase with increasing level of immune suppression $[12,13]$. Adults with HIV-1 not on HAART show a greater risk of presenting with oral lesions and periodontal pockets than adults with HIV-1 receiving HAART. There are indications however, that individuals with HIV1 on long- term HAART medication have a greater risk of oral lesions than their counterparts on short-term medication [14].

Considering the association between HIV-1 infection and dental caries, data have been inconsistent. No difference in dental caries was observed between uninfected-and perinatally exposed HIV uninfected children in Uganda [15]. In contrast, a Nigerian study comparing three groups of preschool children, reported higher caries prevalence in HIV infected- than in HIV uninfected children, whereas no difference was found between HIV uninfected and HIV exposed uninfected, children [6]. A systematic review considering caries in the primary and permanent dentition of children with HIV reported increased risk of caries in the primary- but not in the permanent dentition [16]. Moscicki et al [17] reported on higher caries prevalence in youth with HIV compared with their exposed uninfected counterparts but no difference regarding periodontal disease. Women's Interagency HIV Study (WIHS), including the largest cohort of HIV infected women in the United States, revealed significantly higher caries scores (number of decayed, missed filled teeth, DMFT) in women with HIV compared to HIV negative women [18]. Moreover, reports reveal an increase in caries prevalence following HAART medication among people with HIV $[19,20]$ and suggest that the prevalence of dental caries varies according to the type and length of HAART medication taken [14]. A study in Uganda reported a caries prevalence of above $80 \%$ in HIV positive adults, whereas the corresponding parameters in the general adult population were estimated to $67 \%$. This study also indicated that longer duration of HAART medication associated with increased risk of dental caries [20].

Factors, such as xerostomia, microbial colonization, poor oral hygiene, periodontal disease and carbohydrate diet increase susceptibility to dental caries in HIV infected adults [20]. Kumar et al [11] and Soares et al [21] reported on higher caries experience and higher prevalence of periodontal disease in HIV positive patients of low-compared to high socio-economic status. A question arises as to whether oral diseases are more frequent in the lower- compared to the higher socio-economic status groups of HIV infected people and whether social inequality in oral health status vary between HIV infected-and uninfected individuals.

There is a paucity of studies considering the prevalence and socio-behavioral covariates of dental caries among adults with HIV-1 in Sub Saharan Africa. Previous studies have considered individuals attending HIV care clinics and many have omitted an adequate comparison groups of uninfected individuals. Moreover, studies examining dental caries generate multiple outcome data. An important caveat is avoidance of utilizing multilevel modeling for hierarchical clustered data. Ignoring that tooth surfaces are clustered within teeth and that teeth are clustered within individuals by using classic statistical methods might provide biased regression estimates and underestimated standard errors. 
Purpose

This study aims to assess caries experience in Ugandan mothers according to HIV-1 status, sociobehavioral characteristics and gingival bleeding status and examine whether HIV status modify the association of socio-behavioral covariates with caries experience. Secondly, using multilevel analysis, this study also assessed to what extent site specific caries experience vary between and within individuals.

\section{Methods}

This cross-sectional study uses data from the follow-up of women with HIV-I involved in the efficacy trial of clinical HIV-1 peri-exposure prophylaxis with ritonavir boosted lopinavir- (LPV/r), the ANRS12174 PROMISE- PEP trial (ClinicalTrials.gov, number NCT00640263). The ANRS12174 PROMISE- PEP trial is described in detail in previous papers [22]. The trial conducted between 2009 and 2013, was a multicenter randomized trial including pregnant women with HIV-1, recruited at gestational age of 28-40 weeks at antenatal clinics in four African sites; Ouagadougou, Burkina Faso; East London, South Africa; Mbale, Eastern Uganda; and Lusaka, Zambia. HIV-1 infected pregnant women were referred for further assessment of inclusion criteria and again with their HIV uninfected children for enrolment at day 7 postpartum. Infants were eligible for inclusion if they were: a singleton; breastfed at day seven by their mothers; had a negative HIV-1 DNA PCR blood test and had received any Prevention of-Mother-to-ChildTransmission, PMTCT. Inclusion criteria for mother was age 18 years or older, intention to continue breastfeeding, being HIV-1 infected, and not being eligible for ART (either clinically or because CD4 count $>350$ cells/ $\mu \mathrm{L}$ at that time). All eligible mothers and infants followed the routine PMTCT with antepartum zidovudine (ZDV), intrapartum nevirapine (NVP), zidovudine-lamivudine (ZDV/3TC) for mothers and NVP for infants 7 days postpartum.

In Uganda, 278, seven-day old uninfected children born to HIV-1 infected women were randomized to receive infant prophylaxis (either $3 \mathrm{TC}$ or LPV/r daily) throughout the breastfeeding period from day 7 to 50 weeks. The primary outcome was mother to child HIV-1 transmission, diagnosed at day 7 and 6, 14, 26, 38 and 50 weeks with HIV-1 DNA PCR between 7 days and 50 weeks post-delivery. Findings from the PROMISE- PEP trial showed that infection rates, and clinical and biological severe adverse events did not differ between the two drug regimens suggesting that infant HIV-1 prophylaxis with either drug was not superior as both led to very low rates of HIV-1 postnatal transmission during 50 weeks of breastfeeding [22].

In 2017, 244 out of 278 mothers with HIV-1 infection and their uninfected children were eligible for reenrollment in the follow-up study: the PROMISE-PEP Mechanism Safety study (PROMISE-PEP M\&S ANRS12341). Of the 166 HIV exposed uninfected children (HEU) re-enrolled, 2 were excluded because they had contracted HIV during the follow up period. Thus, $68 \%$ of the eligible cohort of HEU children $(164 / 244)$ and their HIV-1 infected mothers were followed up with 32\% ( $n=112)$ missed due to attrition. A comparison group of 199 HIV unexposed uninfected (HUU) children matched on age and sex, as well as 
their HIV uninfected mothers were recruited from communities located in Mbale, Eastern Uganda, which was the site for the ANRS12174 PROMISE- PEP trial. Of the $199 \mathrm{HUU}$ control children, 19 were excluded due to a positive HIV-1 test result, leaving 181 HUU children and their uninfected mothers enrolled. The present cross-sectional study is based on information from interviews and clinical oral examinations of 164 HIV-1 infected- and 181 HIV-1 uninfected mothers participating in the follow-up study, the PROMISEPEP Mechanism Safety study (PROMISE-PEP M\&S ANRS12341) in 2017.

\section{Interviews with HIV-1 infected mothers and HIV - 1 negative controls}

Trained interviewers performed face-to-face interviews with mothers using semi-structured interviews in one of the local Ugandan languages, Lumasaba. The interview was constructed in English and translated into Lumasaba for use in the field. The schedule had been reviewed previously by project staff for semantic, experiental and conceptual equivalence of the source version. Sensitivity to culture and selection of appropriate words were considered [22, 23]. Mothers responded to questions about themselves and their children. Information was documented on case record forms (CRFs) and electronically with Capture software System (Clinsight) and Epidata program www.epidata.dk for the clinical oral examinations.

Socio-demographic characteristics of caretakers were assessed in terms of level of education, type of income and marital status. Level of education was categorized into 'did not finish primary school (1), end of primary school (2) higher education' (3). Marital status was categorized: 'divorced (1), cohabiting/married (2), single (3), widowed' (4) and recoded into single/divorced/widow (0) and married/cohabiting (1). Mother's behavioral characteristics were assessed in terms of tooth brushing frequency and frequency intake of sugared snacks. Type of income was categorized into (1) no regular income (2) regular income.

\section{Clinical oral examination}

Two experienced and calibrated dental surgeons (NB and MM) performed the oral assessments among the study participants and duplicate full-mouth oral clinical examination among HIV-1 positive and HIV negative mothers not included in the main study. Dental caries was assessed on surface and tooth level (5 surfaces per tooth) in terms of decayed (D), missing (M), and filled (F) surface/teeth (DMFS/DMFT) in accordance with the World Health Organization (WHO) guidelines for field conditions [24]. Each surface was recoded 0 for sound and 1 for caries experience and documented as decayed if it was visually cavitated with the aid of a dental mirror and periodontal probe. A surface was recorded filled when treated and a tooth was recorded missing when extracted due to caries, as confirmed by the participant. To assess gingival bleeding of the individual, the modified community periodontal index (CPI) was used. Each tooth was scored according to the presence or absence of gingival bleeding, using a periodontal probe across the gingival margins of the teeth. An individual score of presence of gingival bleeding was given if a tooth had bleeding on probing. 


\section{Maternal HIV-1 status of HIV-1 uninfected controls}

Mothers in the comparison group were tested for their HIV-1 status using serial and parallel HIV rapid testing with Determine, Stat-Pak and Uni-Gold, three test algorithms as recommended by the Ugandan Ministry of Health [25].

\section{Statistical analysis}

STATA SE 16 (College Station, Texas 77845 USA) was used for data analysis. Cross-tabulation and Chisquare tests for categorical variables were used to assess the crude associations of covariates and outcome variables according to HIV-1 status. Site specific dental caries data has a clustered 3-level hierarchical structure with surfaces (level 1) clustered within tooth (level 2) and teeth clustered within individuals (level 3). Using site specific measures of caries as independent observations and ignoring that observations are clustered and correlated within individual will lead to an underestimation of the standard errors, too narrow confidence intervals and higher type 1 error rates. In this study we first applied ordinary logistic regression on the individual level with aggregated measures of Decayed Missed and Filled Teeth (DMFT) as dichotomized outcome variable (DMFT >0). Socio-behavioral and clinical covariates statistically significantly associated both with HIV-1 status and DMFT were included in the ordinary logistic, and potential confounding variables. Secondly, we applied caries on all observed sites within individual. This was done using a random intercept model (RIM) (random intercepts for teeth and individuals) using mixed effect logistic regression with the melogit package within the Stata program (version SE 16) fitted for caries experience. Due to high correlation of caries on surface level within tooth, a three-level model including random intercepts for both individuals and teeth could not be fitted. Thus, we fitted a two-level model with sites within individuals. Relationships between outcome and covariates were assumed with all the cluster regression lines having a fixed slope and different intercepts. The effect of dependency of caries on sites within individual was assessed by calculating intra class correlation coefficients (ICC). The ICC expresses variations between individuals as a proportion of the total variance. ICC varies from 0 , which implies that caries is independent within individuals to 1 indicating no variation of caries within individual. We applied a likelihood-ratio test to test if ICC equals 0 . A statistical significance implies that the multilevel model is preferable. P-values less than 0.05 were considered statistically significant.

\section{Results}

Cohen's Kappa values for intra- and inter-rater reliability were 0.7 (95\% Cl: $0.5-0.9)$ and 0.6 (95\% Cl: $0.4-$ $0.8)$, respectively.

Table 1 provides descriptive analyses of independent and dependent variables at the individual level. A total of $164 \mathrm{HIV}$ infected and $181 \mathrm{HIV}$ uninfected mothers were interviewed and examined for dental caries and gingival bleeding. A total of $162(50 \%)$ was younger than 33 years. Prevalence of individuals with decayed surfaces (i.e. proportion of participants with DMFS $>0$ ) was $74 . \%$. Corresponding prevalence figure for decayed teeth (i.e. proportion of individuals with DMFT >0) was 61.\%. 
Table 1

Socio-demographic- clinical and behavioral characteristics of mothers by HIV-1 status at follow-up in the Ugandan part of the PROMISE-PEP-M\&S study.

\begin{tabular}{|c|c|c|c|}
\hline & $\begin{array}{l}\text { HIV-infected } \\
(n=164)\end{array}$ & $\begin{array}{l}\text { HIV- un-infected } \\
(n=181)\end{array}$ & $\begin{array}{l}\text { Total Individual } \\
(n=345)\end{array}$ \\
\hline$T$ & $\%(n)$ & $\%(n)$ & $\%(n)$ \\
\hline \multicolumn{4}{|l|}{ Mother /caretaker characteristics } \\
\hline \multicolumn{4}{|l|}{ Age } \\
\hline $18-32 y r$ & $40(58)$ & $58(104)$ & $50(162)$ \\
\hline $33+$ & $60(90) * \star$ & $42(76)$ & $50(166)$ \\
\hline \multicolumn{4}{|l|}{ Toothbrush } \\
\hline Once a day or less & $59(97)$ & $44(78)$ & $51(175)$ \\
\hline More than once a day & $40(66)$ & $56(99) \star \star$ & $48(165)$ \\
\hline \multicolumn{4}{|l|}{ Sugared snacks } \\
\hline At most occasionally & $92(151)$ & $88(159)$ & $90(310)$ \\
\hline At last once a day & $8(13)$ & $12(21)$ & $10(34)$ \\
\hline \multicolumn{4}{|l|}{ Marital status } \\
\hline Single/divorced, widow & $26(43)$ & $18(33)$ & $22(76)$ \\
\hline Married/cohabiting & $74(121)$ & $82(147)$ & $78(268)$ \\
\hline \multicolumn{4}{|l|}{ Type of income } \\
\hline No regular & $60(98)$ & $72(130)$ & $66(228)$ \\
\hline Regular & $49(65)$ & $28(51) *$ & $34(116)$ \\
\hline \multicolumn{4}{|l|}{ Educational level } \\
\hline Primary school & $38(54)$ & $40(65)$ & $38(119)$ \\
\hline End of primary school & $20(28)$ & $22(37)$ & $21(65)$ \\
\hline Middle school/high school/college & $42(59)$ & $38(63)$ & $40(122)$ \\
\hline \multicolumn{4}{|l|}{$D M F T$} \\
\hline $\mathrm{DMFT}=0$ & $19(31)$ & $29(52)$ & $24(83)$ \\
\hline \multicolumn{4}{|l|}{${ }^{*} p<0.05 ; \chi^{2}$ test, ${ }^{* *} p<0.001 ; \chi^{2}$ test } \\
\hline Numbers do not add to 345 in the & us cells due & missing respons & \\
\hline
\end{tabular}




\begin{tabular}{|llll|}
\hline & $\begin{array}{l}\text { HIV- infected } \\
(\mathbf{n}=164)\end{array}$ & $\begin{array}{l}\text { HIV- un-infected } \\
(\mathbf{n}=181)\end{array}$ & $\begin{array}{l}\text { Total Individual } \\
(\mathbf{n}=345)\end{array}$ \\
\hline DMFT $>0$ & $81(133)$ & $71(129)$ & $3(4.3)$ \\
\hline DMFT $\geq 1$ & $5(5.3)$ & $3(3.2)$ & $76(264)$ \\
\hline DT $>0$ & $76.2(125)$ & $46(84)$ & $61(209)$ \\
\hline DMFS & & & $26(91)$ \\
\hline DMFS $=0$ & $22(37)$ & $54(29.8)$ & $74(254)$ \\
\hline DMFS $>0$ & $77(127)$ & $70(127)$ & $62(213)$ \\
\hline DS $>0$ & $93(51)$ & $120(73.2)$ & $26(90)$ \\
\hline Mothers health & & & $74(254)$ \\
\hline Bad/fair & $35(57)$ & $18(33)$ & $46(160)$ \\
\hline Good/very good & $82(147)$ & $65(107) * *$ & $54(185)$ \\
\hline Presence of gingivitis & & & \\
\hline No & $35(58)$ & $54(102)$ & $(79) * *$ \\
\hline Yes & $64(105)$ & & \\
\hline * $p<0.05 ; \chi^{2}$ test, ${ }^{* *} p<0.001 ; \chi^{2}$ test & & & \\
\hline Numbers do not add to 345 in the various cells due to missing responses & \\
\hline
\end{tabular}

The distribution of participants' socio-demographic-, behavioral and clinical characteristics according to mothers' HIV-1 status are presented in Table 1. Age, marital status, type of income, mothers' perceived health, gingival bleeding, and caries experience varied statistically significantly (all $p<0.05$ ) according to HIV-1 status. Mothers with HIV-1 were more frequently older than 33 years of age, were less likely to brush teeth more than once a day, less likely to be married and more likely to have regular income compared to HIV negative mothers. Mothers with HIV-1 were more likely to express bad perceptions of health and to present with gingival bleeding.

Table 2 depict percentage distribution, odds ratios (OR) with 95\% confidence intervals for dental caries experience (DMFT $>0 / D M F T \geq 1$ ) by HIV-1 status and socio-behavioral and clinical covariates. Significant crude association occurred between caries experience and HIV-1 status, educational level, perceived health and presence of gingival bleeding. Caries experience differed statistically significantly between HIV-1 infected and non-infected mothers with $81 \%$ and $71 \%$ having DMFT $>0$, respectively. Moreover, $82 \%$ versus $69 \%$ of mothers with and without presence of gingival bleeding presented with DMFT $>0$. Ordinary adjusted logistic regression analysis revealed that HIV-1 infected mothers were more likely than their HIV-1 negative counterparts to experience DMFT >0 (OR $=1.8,95 \% \mathrm{Cl} 1.0-3.4)$. Mothers 
with higher education (high school and college) were more likely to experience caries compared to their lower educated counterparts $(\mathrm{OR}=1.9,95 \% \mathrm{Cl}: 1.0-3.6)$. Moreover, mothers presenting with gingival bleeding were more likely to present with caries experience $(\mathrm{OR}=2.1,95 \% \mathrm{Cl}: 1.1-3.8)$. Two-way interactions terms between HIV-1 status and each of the socio-demographic, behavioral and clinical covariates were added to the regression model after adjustment for the main effect of the variables included in the multiplicative constructs. No significant interactions were observed. 
Table 2

Mothers' dental caries experience according to individual related factors in terms of HIV status, socio-demographic - and behavioral/clinical characteristics. Adjusted ordinary logistic regression $(n=345)$

\begin{tabular}{|c|c|c|}
\hline Mothers characteristics & Cross tabulation & $\begin{array}{l}\text { Adjusted Ordinary logistic regression } \\
\text { DMFT >0 }\end{array}$ \\
\hline HIV-1 Status & $\%(n)$ & OR $(95 \% \mathrm{Cl})^{e}$ \\
\hline HIV-1 uninfected & $71(129)$ & 1 \\
\hline HIV-1 infected & $81(133)$ * & $1.8(1.0-3.4)$ \\
\hline \multicolumn{3}{|l|}{ Age } \\
\hline $18-32$ & $72(117)$ & 1 \\
\hline $33+$ & 79 (132) & $1.5(0.8-2.7)$ \\
\hline \multicolumn{3}{|l|}{ Marital status } \\
\hline Single/divorced, widow & $79(60)$ & 1 \\
\hline Married/cohabiting & $75(202)$ & $1.0(0.6-2.1)$ \\
\hline \multicolumn{3}{|l|}{ Type of income } \\
\hline No regular & $67(175)$ & 1 \\
\hline Regular & $33(86)$ & $0.9(0.5-1.8)$ \\
\hline \multicolumn{3}{|l|}{ Educational level } \\
\hline Primary school & $74(88)$ & 1 \\
\hline End of primary school & $71(46)$ & $0.8(0.4-1.8)$ \\
\hline Middle school/high school/college & $82(100)$ & $1.9(1.0-3.6)$ \\
\hline \multicolumn{3}{|l|}{ Gingivitis } \\
\hline Not present & $69(110)$ & 1 \\
\hline Present & $82(152) * \star$ & $2.1(1.1-3.8)$ \\
\hline \multicolumn{3}{|c|}{${ }^{*} p<0.05 ; \chi^{2}$ test, ${ }^{* *} p<0.001 ; \chi^{2}$ test, ${ }^{e}$ odds ratio $95 \%$ confidence interval } \\
\hline Numbers do not add to 345 in the va & ious cells due to $\mathrm{m}$ & ssing responses \\
\hline
\end{tabular}

Table 3 depicts the fixed effects on site specific caries of mothers' HIV-1 status, socio-demographic factors, gingival bleeding, tooth groups and tooth surfaces. In the multilevel logistic regression analysis, a total of 46095 surfaces and a mean number of 158 (range 140-160) surfaces per individual of were analyzed. 
Table 3

Mothers' dental caries experience according to HIV-1 status, age, marital status, education, gingivitis, tooth groups and surfaces. Multilevel analysis (melogit) allowing for clustering at individual level ( $\mathrm{n}=$ 46095).

\section{Mothers characteristics}

HIV-1 Status

HIV-1 uninfected

HIV-1 infected

Age

$18-32$

$33+$

Marital status

Single/divorced, widow

Married/cohabiting

Type of income

No regular

Regular

Educational level

Primary school

End of primary school

Middle school/high school/college

Gingivitis

Not present

Present

Tooth group

Premolars

Molars- maxilla

Molars mandibular

Values are person specific estimates adjusted for tooth group, surfaces and individual level covariates calculated using a melogit multilevel regression model with a random intercept

\section{Adjusted melogit DMFS $>0$}

OR $(95 \% \mathrm{Cl}){ }^{\mathrm{e}}$

1

$1.4(0.8-2.2)$

1

$1.8(1.1-2.8)$

1

$1.1(0.6-2.0)$

1

$0.9(0.5-1.6)$

1

$0.8(0.4-1.4)$

$1.2(0.7-2.1)$

1

$2.0(1.2-3.2)$

1

$7.4(6.6-8.3)$

$17.0(15.2-18.2)$

e Odds ratio (95\% confidence interval) 


\section{Mothers characteristics}

Incisor

Surfaces

Buccal

Distal

Mesial

Occlusal

Palatal/lingual

ICC within subject (adjusted for covariates)

Random effect

Model fit

AIC

\section{Adjusted melogit DMFS $>0$}

$0.5(0.4-0.7)$
1

$1.1(0.9-1.2)$

$1.1(0.9-1.2)$

$0.9(0.8-1.1)$

$2.7(2.4-3.0)$

$0.54(0.48-0.59)$

$3.8(3.0-4.8)$

4297.60; $\mathrm{P}<0.0000$

18498.95

Values are person specific estimates adjusted for tooth group, surfaces and individual level covariates calculated using a melogit multilevel regression model with a random intercept

eOdds ratio (95\% confidence interval)

The multilevel model revealed significant positive associations between caries and women older than 33 years of age $(\mathrm{OR}=8.1,95 \% \mathrm{Cl}: 1.1-2.8)$, and between caries and presence of gingival bleeding (OR = $2.0,95 \% \mathrm{Cl}: 1.2-3.2)$. Compared to premolar teeth, caries experience was more likely to occur in maxillary molars ( $\mathrm{OR}=7.4: 95 \% \mathrm{Cl}: 6.6-8.3)$, mandibular molars $(\mathrm{OR}=17.0,95 \% \mathrm{Cl}: 15.3-18.2)$ and less likely to occur in incisors $(\mathrm{OR}=0.55,95 \% \mathrm{Cl}: 0.46-0.66)$. Compared to palatal/lingual surfaces caries experience was more likely $(\mathrm{OR}=2.7,95 \% \mathrm{Cl} 2.4-3.0)$ to occur at the buccal surface. The ICC estimate for individuals (level 3 ) amounted to 0.54 (95\% Cl $0.48-0.59$ ), indicating that $54 \%$ and $46 \%$ of the variance in caries experience was attributable to variation between individuals and within individuals, respectively. The likelihood ratio test confirmed that ICC was different from zero and that a two-level model is justified and an appropriate approach to the present data $(p=0.0000)$.

\section{Discussion}

This study is among the first to report on caries experience in HIV- 1 infected mothers as compared to HIV1 negative controls living in a similar non-occidental socio-cultural setting. The prevalence of caries experience, untreated caries and gingival bleeding was high among both HIV-1 infected- and uninfected mothers amounting to $50 \%$ or above of the study population. Ordinary logistic regression with aggregated outcome measures of caries experience revealed significant odds for mothers who were HIV-1 infected, older aged, having higher education and gingival bleeding, whereas multilevel analyses of site specific 
caries revealed significant odds for mother's age and gingival bleeding status. Socio-behavioral inequalities in dental caries experience were less marked, nor did socio-behavioral inequalities in dental caries differ between HIV-1 infected- and uninfected mothers. This supports evidence that the HIV-1 epidemic in low-income countries seem to be more generalized across the socio-economic spectrum, suggesting that HIV-1 positive and negative mothers are more socio-economically comparable [26]. We conclude that a multilevel random intercept model is appropriate and efficient for analyses of dental caries data in the context of HIV-1 infected Ugandan mothers. It improves the standard logistic regression approach by inclusion of covariates at site- and tooth level, A significant amount of variability was attributable to the between individual level implying that carious surfaces and teeth were more highly correlated within than between mothers. Although we aimed to fit a 3- level model with surfaces nested within teeth and teeth nested within individuals, there was hardly any variation of caries at tooth level.

Major strengths of this study are the use of novel statistical methods accounting for dependency in observations within individual as well as strict characterization of HIV-1 status and use of community controls [27]. Whereas models for dependent observations are well established in medical research, their application are limited in dentistry [28-30]. Previous studies applying a multilevel approach have been performed, most commonly, in the field of periodontology [31]. Yet, few studies are available utilizing multilevel analysis with hierarchical structured caries data [32]. A benefit of multilevel analysis is the possibility to separate variance estimates at different levels, allowing for estimation of intra class correlation at the individual and tooth level. This offers the advantage of more information of caries patterns within an individual. This study utilized both ordinary- and mixed effect logistic regression models, thus confirming inequalities in mothers' caries experience at individual and site specific levels. Estimates from ordinary logistic regression models with aggregate caries outcome measures define differences in caries experience among population groups having different characteristics. A mixed model logistic regression approach provides person specific estimates or change in odds for a specific mother according to her changes in characteristics. Despite difference in interpretation of the models and the estimates from the models, ordinary logistic- and mixed model logistic regression revealed comparable results. Some limitations should be considered, when interpreting our findings. The lack of statistically significant association of HIV-1 status with caries experience might be attributed to limited statistical power as mixed and random effect models are computationally intensive. Missing data and loss to follow-up among HIV-1 infected mothers might limit the interpretation of the findings as the present analyses might suffer from being underpowered. It is possible that caries prevalence in HIV-1 infected mothers is underestimated since only women passively followed up were included in the analyses and those who might have died before follow-up examination were not part of the group analyzed.

The prevalence of dental caries was high in this study population and matched previous estimates in the general Ugandan adult population amounting to 67\% [33]. Mothers living with HIV-1 presented with a caries prevalence of $81 \%$, being in accordance with previous estimates in comparable groups of Ugandan adults amounting to $83 \%$ [20]. HIV-1 infected mothers seemed to be at higher risk of developing dental caries compared to the uninfected controls. Although a specific measure of duration of HAART 
medication was not available, in this study, HIV-1 infected mothers who were follow-up participants from the PROMISE PEP trial had been on systematic HAART medication for more than 6 years. Studies focusing caries among HIV- 1 infected adults on HAART have not yet identified any specific trend $[14,20]$. However, a recent Ugandan study reported on increased prevalence of dental caries according to longer duration of HAART medication [20]. Independent of mothers' HIV-1 status, findings from multilevel analysis indicated higher odds of caries in maxillary and mandibular molar teeth and lower odds in incisor teeth as compared to premolars. This supports well established evidence that caries varies according to different groups of teeth within individuals [34].

It is important to discuss the unexpected positive association between high education and caries experience as depicted in Table 3. This is contrary to previous findings among Ugandan adults living with HIV-1 showing an expected negative relationship between education level and dental caries [20]. In support of the present findings are studies from Africa showing higher prevalence of dental caries in people from less poor- compared to very poor households [35]. It is possible that the coding with only three categories have failed to capture the effect of education very well. It is also possible that this association reflects a higher $\mathrm{F}$ component among higher than lower educated since the highly educated are those most frequently using dental care services.

A positive association between gingival bleeding and dental caries accords with other findings reporting on higher probability of dental caries in people with poor than with good oral hygiene [32,36]. While it appears that HAART reduces saliva flow with above $80 \%$ of the prescribed HAART medications causing xerostomia [14], HIV-1 infection might also decrease saliva irrespective of medication [14, 37]. Salivary gland hypofunction, xerostomia and HAART medication are among the most important risk factors of dental caries and periodontal disease in HIV-1 infected people.

\section{Conclusions}

In summary, this study suggests that dental caries prevalence is higher in HIV-1 infected than in HIV-1 uninfected Ugandan mothers and varies with mothers, age, education and clinically recoded gingival inflammation. Socio-demographic differences in dental caries did not vary by HIV-1 status. We found that a multilevel random intercept model is appropriate and efficient for analyses of dental caries data in the context of HIV-1 infected and uninfected mothers. Based on the present findings we recommend that oral care- and preventive strategies are included into HIV-1 treatment programs.

\section{Declarations}

\section{Ethics approval and consent to participate}

Ethical clearance was obtained from the School of Medicine Research and Ethics committee, Makerere University (SOMREC)-REC - 030, Uganda National Council of Science and Technology (UNCST)-HS 2373 
and the Regional Committee for Health and Medical Research (REK) - 2017/760/REC sør- $\varnothing$ st C from Norway.

\section{Consent for publication}

Not applicable

\section{Availability of data and materials}

The datasets used and/or analyzed during the current study are available from the corresponding author on reasonable request.

\section{Competing interests}

The authors declare no competing interests

\section{Funding}

This study used data collected in the context of the ANRS 12174 clinical trial and 12341 study funded by the French National Institute of Health and Medical Research - National Agency for Research on AIDS and Viral Hepatitis (Inserm-ANRS), the European Developing Countries Clinical Trials Partnership (EDCTP; grant number CT.2006.33020.004), the Research Council of Norway (GlobVac grant number 183600) and the Total Foundation. NB benefited from a postdoctoral research position funded by the Department of Clinical Dentistry, Faculty of Medicine, University of Bergen.

\section{Author contributions}

NB, AN, IMSE JKT, LTF and SAL made contributions to conception and design of the study. NB, AN and JKT contributed to data acquisition. NB, AN, IMSE, JKT, LTF and SAL analyzed, interpreted, drafted, and gave final approval to this version of the manuscript.

\section{Acknowledgements}

This study used data collected in the context of the ANRS 12174 clinical trial and 12341 study. We are grateful to the ANRS 12174 and 12341 for sharing data and giving us the opportunity to carry out oral assessments.

\section{Abbreviations}

AIDS
Acquired immune deficiency syndrome

Antiretroviral therapy 
Human deficiency virus

HEU

HIV exposed uninfected

HUU

HIV unexposed uninfected

ICC

Inter class correlation

IQR

Interquartile range

$\mathrm{LPV} / \mathrm{r}$

Lopinavir/ritonavir

NVP

Niverapine

OR

Odds ratio

OLR

Ordinary logistic regression

PMTCT

Prevention of mother to child transmission

PROMISE-PEP

Promoting infant health and nutrition in Sub-Saharan Africa: Safety and efficacy of infant Peri-Exposure Prophylaxis

PROMISE-PEP-M\&S Promoting infant health and nutrition in Sub-Saharan Africa: Safety and efficacy of infant Peri-Exposure Prophylaxis to prevent HIV-1 transmission by breastfeeding-Mechanisms \& Safety

RIM Random intercept model

WHO World Health Organization

WIHS Womens interagency HIV Study 


\section{References}

1. UNAIDS: Global HIV \& AIDS statistics-2019 fact sheet: UNAIDS; 2019.

2. Challacombe SJ: Global oral inequalities in HIV infection. Oral Dis 2016, Suppl 1:35-41.

3. El Howati A, Tappuni A: Systematic review of the changing pattern of the oral manifestations of HIV. J Investig Clin Dent 2018; 9:e12351.

4. de Aguiar Ribeiro A, Portela MB, de Souza IP: The oral health of HIV-infected Brazilian children. Int J Paediatr Dent 2013; 23:359-65.

5. Shiboski HC, Yao ST-J, Russell IJ, Ryder BM, Van Dyke RR, Seage RG, Moscicki RA-B: The association between oral disease and type of antiretroviral therapy among perinatally HIV-infected youth. AIDS 2018; 32:2497-2505.

6. Coker M, El-Kamary S, Enwonwu C, Blattner W, Langenberg P, Mongodin E, Akhigbe P, Obuekwe O, Omoigberale A, Charurat M: Perinatal HIV Infection and Exposure and their Association with Dental Caries in Nigerian Children. Pediatr Infect Dis J 2017;37:57-65.

7. Griffen AL, Thompson ZA, Beall CJ, Lilly EA, Granada C, Treas KD, DuBois KR, Hashmi SB, Mukherjee C, Gilliland AE et al: Significant effect of HIV/HAART on oral microbiota using multivariate analysis. Sci Rep 2019; 9:1-9.

8. Ryder MI, Yao T-J, Russell JS, Moscicki A-B, Shiboski CH,for the Pediatric HIV/AIDS Cohort Study: Prevalence of periodontal diseases in a multicenter cohort of perinatally HIV-infected and HIVexposed and uninfected youth. J Clin Periodontol 2017; 44:2-12.

9. Pattrapornnan P, DeRouen TA: Associations of periodontitis and oral manifestations with CD4 counts in HIV-pregnant women in Thailand. Oral Surg. Oral Med. Oral Pathol. Oral Radiol 2013; 116:306-12.

10. Ranganathan K, Hemalatha R: Oral Lesions in HIV Infection in Developing Countries: an Overview. Adv Dent Res 2006; 19:63-8.

11. Kumar S, Mishra P, Warhekar S, Airen B, Jain D, Godha S: Oral Health Status and Oromucosal Lesions in Patients Living with HIV/AIDS in India: A Comparative Study. AIDS Res Treat 2014;480247

12. Okoh M, Saheeb B, Agbelusi G, Omoregie F: Relationships between CD4+ Counts and the Presence of Oral Lesions in Human Immunodeficiency Virus Positive Women in Nigeria. Ann Med Health Sci Res 2014; 4:572-77.

13. Naidu GS, Thakur R, Singh AK, Rajbhandary S, Mishra RK, Sagtani A: Oral lesions and immune status of HIV infected adults from eastern Nepal. J Clin Exp Dent 2013; 5:e1-7.

14. Nittayananta W, Talungchit S, Jaruratanasirikul S, Silpapojakul K, Chayakul P, Nilmanat A, Pruphetkaew N: Effects of long-term use of HAART on oral health status of HIV-infected subjects. J Oral Pathol Med 2010; 39:397-406. 
15. Birungi N, Fadnes LT, Engebretsen IMS, Lie SA, Tumwine JK, Åstrøm AN, for the A, study group: Caries experience and oral health related quality of life in a cohort of Ugandan HIV-1 exposed uninfected children compared with a matched cohort of HIV unexposed uninfected children. BMC Public Health 2020; 20:423.

16. Oliveira CAGR, Tannure PN, de Souza IPR, Maia LC, Portela MB, Castro GFBdA: Is dental caries experience increased in HIV-infected children and adolescents? A meta-analysis. Acta Odontol Scand 2014; 73:481-87.

17. Moscicki AB, Yao TJ, Ryder MI, Russell JS, Dominy SS, Patel K, McKenna M, Van Dyke RB, Seage GR, 3rd, Hazra R: The Burden of Oral Disease among Perinatally HIV-Infected and HIV-Exposed Uninfected Youth. PLoS One 2016; 11:e0156459.

18. Phelan J, Mulligan R, Nelson E, Brunelle J, Alves M, Navazesh M, Greenspan D: Dental caries in HIVseropositive women. J Dent Res 2004; 83:869-873.

19. Schmidt-Westhausen AM, Priepke F, Bergmann FJ, Reichart PA: Decline in the rate of oral opportunistic infections following introduction of highly active antiretroviral therapy. J Oral Pathol Med 2000; 29:336-41.

20. Kalanzi D, Mayanja-Kizza H, Nakanjako D, Mwesigwa CL, Ssenyonga R, Amaechi BT: Prevalence and factors associated with dental caries in patients attending an HIV care clinic in Uganda: a cross sectional study. BMC Oral Health 2019; 19:159-58.

21. Soares GB, Garbin CAS, Moimaz SAS, Garbin AJI: Oral health status of people living with HIV/AIDS attending a specialized service in Brazil: Spec Care Dentist 2014; 34:176-184.

22. Nagot N, Kankasa C, Tumwine JK, Meda N, Hofmeyr GJ, Vallo R, Mwiya M, Kwagala M, Traore H, Sunday $A$ et al: Extended pre-exposure prophylaxis with lopinavir-ritonavir versus lamivudine to prevent HIV-1 transmission through breastfeeding up to 50 weeks in infants in Africa (ANRS 12174): a randomised controlled trial. Lancet 2016; 387:566-573.

23. Birungi N, Fadnes LT, Okullo I, Kasangaki A, Nankabirwa V, Ndeezi G, Tumwine JK, Tylleskar T, Lie SA, Astrom AN: Effect of Breastfeeding Promotion on Early Childhood Caries and Breastfeeding Duration among 5 Year Old Children in Eastern Uganda: A Cluster Randomized Trial. PLoS One 2015; 10:e0125352.

24. Who: Oral Health Surveys : Basic Methods (5th Edition). Albany, NY, USA: World Health Organization; 2013.

25. Galiwango RM, Musoke R, Lubyayi L, Ssekubugu R, Kalibbala S, Ssekweyama V, Mirembe V, Nakigozi G, Reynolds SJ, Serwadda D et al: Evaluation of current rapid HIV test algorithms in Rakai, Uganda. J Virol Methods 2013; 192:25-27.

26. Filteau S: The HIV-exposed, uninfected African child L'enfant africain exposé au VIH mais non infecté: une revue El VIH expuesto, el niño Africano no infectado: revisión. Trop Med Int Health 2009; 14:276-87.

27. Fleming PS, Koletsi D, Polychronopoulou A, Eliades T, Pandis N: Are clustering effects accounted for in statistical analysis in leading dental specialty journals? J Dent 2013; 41:265-70. 
28. Gülcan F, Ekbäck G, Ordell S, Lie SA, Åstrøm AN: Social predictors of less frequent dental attendance over time among older people: population-averaged and person-specific estimates. Community Dent Oral Epidemiol 2016; 44:263-73.

29. Dobloug A, Grytten J, Holst D: Dentist-specific variation in diagnosis of caries - a multilevel analysis. Community Dent Oral Epidemiol 2014; 42:185-91.

30. André Kramer A-C, Pivodic A, Hakeberg M, Östberg A-L: Multilevel Analysis of Dental Caries in Swedish Children and Adolescents in Relation to Socioeconomic Status. Caries Res 2019; 53:96-106.

31. Bunæs DF, Lie SA, Åstrøm AN, Mustafa K, Leknes KN: Site-specific treatment outcome in smokers following 12 months of supportive periodontal therapy. J Clin Periodontol 2016; 43:1086-1093.

32. Masood M, Masood Y, Newton JT: The Clustering Effects of Surfaces within the Tooth and Teeth within Individuals. J Dent Res 2014; 94:281-88.

33. Kutesa A, Kasangaki A, Nkamba M, Muwazi L, Okullo I, Rwenyonyi CM: Prevalence and factors associated with dental caries among children and adults in selected districts in Uganda. Afr Health Sci 2015; 15:1302-07.

34. Batchelor PA, Sheiham A: Grouping of tooth surfaces by susceptibility to caries: a study in 5-16 year-old children. BMC Oral Health 2004; 4:2.

35. Nurelhuda NM, Trovik TA, Ali RW, Ahmed MF: Oral health status of 12-year-old school children in Khartoum state, the Sudan; a school-based survey. BMC Oral Health 2009; 9:15.

36. Simangwa LD, Åstrøm AN, Johansson A, Minja IK, Johansson A-K: Oral diseases and oral health related behaviors in adolescents living in Maasai population areas of Tanzania: a cross-sectional study. BMC Pediatr 2019; 19:275-14.

37. Liberali SA, Coates EA, Freeman AD, Logan RM, Jamieson L, Mejia G: Oral conditions and their social impact among HIV dental patients, 18 years on. Aust Dent J 2013; 58:18-25. 